\title{
Der Schweizer Wald und seine Biodiversität: LiDAR ermöglicht neue Waldstrukturanalysen
}

Florian Zellweger Kurt Bollmann
Eidgenössische Forschungsanstalt für Wald Schnee und Landschaft und Waldökologie, ETH Zürich (CH)*

Eidgenössische Forschungsanstalt für Wald, Schnee und Landschaft $(\mathrm{CH})$

\section{Der Schweizer Wald und seine Biodiversität: LiDAR ermöglicht neue Wald- strukturanalysen}

\begin{abstract}
Biodiversitätskarten und bestandesspezifische Empfehlungen für Habitataufwertungen sind für den Forstdienst oft nur beschränkt verfügbar, obwohl solche Informationen für eine multifunktionale Waldbewirtschaftung sehr nützlich sind. Für diesen Artikel kombinierten wir aus national verfügbaren LiDAR-Daten abgeleitete Waldstrukturparameter mit Daten zu Klima, Topografie und Boden- $\mathrm{pH}$, um die Artenvielfalt von verschiedenen Artengruppen im Schweizer Wald auf zwei Skalen zu analysieren und räumlich vorherzusagen. Auf der Bestandesskala $\left(900 \mathrm{~m}^{2}\right)$ analysierten wir die Artenvielfalt von Gefässpflanzen, Moosen und Landschnecken und nutzten gleichzeitig Daten aus dem Landesforstinventar, um potenzielle Zielvariablen für Habitataufwertungen abzuleiten. Auf der Landschaftsskala $\left(1 \mathrm{~km}^{2}\right)$ analysierten wir die Artenvielfalt von Gefässpflanzen, Tagfaltern und Brutvögeln, die eine starke Bindung an den Wald haben. Die aus LiDAR abgeleiteten Waldstrukturparameter waren bei allen Artengruppen wichtige Prädiktoren für die Artenvielfalt. Die verschiedenen Artengruppen zeigten unterschiedliche räumliche Verbreitungsmuster, und ihre Verbreitung überlappte sich nur begrenzt. Die Artenvielfalt der für den Wald typischen Landschnecken beispielsweise nahm mit zunehmendem Anteil von Bäumen der Gattungen Fraxinus, Tilia, Ulmus und Acer zu, ebenso mit zunehmendem Deckungsgrad der Baumschicht und zunehmendem Totholzvolumen. Bei den Tagfaltern hingegen nahm die Artenvielfalt mit zunehmender Heterogenität in der Unterschicht zu. Diese Studie zeigt, dass wichtige Strukturmerkmale für die Waldbiodiversität mit LiDAR flächendeckend über grosse Gebiete erfasst werden können. Damit liefert die steigende Verfügbarkeit von LiDAR-Daten nützliche Informationen für die Erhaltung und Förderung der Biodiversität im multifunktionalen Waldbau.
\end{abstract}

Keywords: biodiversity, forest structure, habitat quality, LiDAR, remote sensing, species diversity doi: 10.3188/szf.2017.0142

*Zürcherstrasse 111, CH-8903 Birmensdorf, E-Mail florian.zellweger@wsl.ch

W älder sind aufgrund ihrer räumlichen Struktur von besonderer Bedeutung für die Biodiversität (MacArthur \& MacArthur 1961). Der Zusammenhang zwischen Waldstruktur und Waldbiodiversität beruht darauf, dass die vertikale und horizontale Anordnung der Pflanzen sowie deren Entwicklung einen starken Einfluss auf die Standortfaktoren und die ökologischen Prozesse im Wald haben (z.B. Licht, Mikroklima, Verjüngung) und die Verfügbarkeit und Diversität von Nischen und Ressourcen beeinflussen. Ein reichhaltig strukturierter Wald bietet somit eine grosse Vielfalt an Nischen und Habitatelementen, zum Beispiel Nist- und Schlafplätze oder Schutz- und Versteckmöglichkeiten (Hunter 1999, Bollmann et al 2009). Deshalb ist die Förderung von strukturellen Eigenschaften und deren Komplexität ein geeignetes Instrument für die Erhaltung und Förderung der bio- logischen Vielfalt im multifunktionalen Waldbau (Bollmann et al 2009, Imesch et al 2015, Seibold et al 2015, Guénette \& Villard 2005, Lindenmayer et al 2006).

Allerdings ist bei der Förderung einer Art oder Artengruppe immer auch auf Zielkonflikte zu achten, damit Förderungsmassnahmen möglichst keine negativen Folgen für andere Arten haben. Für solche Abwägungen braucht es verlässliche Informationen zu den art- oder artengruppenspezifischen Lebensraumanforderungen, und es empfiehlt sich eine räumliche Priorisierung, die sich an der Verbreitung geeigneter Habitate möglichst vieler Arten oder Artengruppen orientiert. Herkömmliche Felderhebungen zur Waldstruktur können hilfreiche Informationen zur Habitatqualität liefern. Solche Erhebungen sind jedoch aufwendig und räumlich oft auf Stichprobenflächen begrenzt, was eine flächendeckende 
Evaluierung erschwert. Fernerkundungsmethoden wie das flugzeuggetragene Laserscanning, auch Light Detection and Ranging (LiDAR) genannt, können diesbezüglich Abhilfe schaffen, weil solche Erhebungen flächendeckend hochaufgelöste und verlässliche Angaben zur dreidimensionalen Struktur des Waldes liefern (Gachet \& Junod 2008, Morsdorf 2011). Mittels LiDAR können nicht nur waldbauliche Parameter wie die Baum- und Bestandeshöhe, die vertikale Schichtung, der mittlere Brusthöhendurchmesser, die Stammzahl oder das stehende Holzvolumen flächendeckend abgeschätzt werden (Næsset 2002), sondern auch Kenngrössen zur Analyse und Vorhersage von Artenvielfalt im Wald (Zellweger et al 2013, Davies \& Asner 2014).

Im vorliegenden Artikel werden Waldstrukturparameter aus LiDAR-Daten hergeleitet und in Kombination mit Parametern zu Klima, Topografie und Boden-pH mit Artnachweisen aus dem Biodiversitätsmonitoring Schweiz (BDM) in Beziehung gesetzt, um die Vielfalt von verschiedenen Artengruppen (Gefässpflanzen, Moose, Landschnecken, Tagfalter, Brutvögel) im Schweizer Wald auf der Bestandes- und der Landschaftsebene räumlich vorherzusagen. Um wichtige Strukturparameter, die im Rahmen der Waldbewirtschaftung beeinflusst werden können, zu identifizieren, werden die Artnachweise zudem mit Daten aus dem Landesforstinventar (LFI) ausgewertet. Der Artikel basiert auf den Beiträgen Zellweger et al (2015) und Zellweger et al (2016).

\section{Material und Methoden}

\section{Artdaten auf Bestandesebene}

Als Grundlage für die Analysen auf der Bestandesebene dienten Nachweise von Gefässpflanzen, Moosen und Landschnecken, die im Rahmen des BDM auf $10 \mathrm{~m}^{2}$ grossen Stichprobenflächen erhoben und unter dem Indikator Z9 (Artenvielfalt in Lebensräumen) zusammengefasst werden (Weber et al 2004, Koordinationsstelle BDM 20081). Berücksichtigt wurden Daten auf Stichprobenflächen im Wald aus den Jahren 2004 bis 2008, wo gleichzeitig auch Daten aus dem LFI vorhanden waren. Für Gefässpflanzen und Moose lagen je 410 Stichprobenflächen vor, für Landschnecken 406. Als Messgrösse für Artenvielfalt diente die Artendichte, d.h. die Anzahl Arten pro Probefläche. Neben der allgemeinen Artenvielfalt haben wir auch die Vielfalt von für den Wald typischen Arten analysiert (nachfolgend Waldarten genannt). Für die Klassierung der Waldarten haben wir Artengruppenexperten befragt und die Artnachweise der nationalen Artenzentren konsultiert. ${ }^{2}$ Generell klassierten wir auf der Bestandesebene eine Art als Waldart, wenn die Artnachweise zu 50\% oder mehr aus dem Wald stammten.

\section{Artdaten auf Landschaftsebene}

Für die Analyse auf der Landschaftsebene verwendeten wir BDM-Daten zum Vorkommen von Gefässpflanzen, Tagfaltern und Brutvögeln aus $1 \mathrm{~km}^{2}$ grossen Stichprobenquadraten (BDM-Indikator Z7), die mindestens zu 30\% bewaldet sind. Die Artnachweise stammen aus den Jahren 2004 bis 2008 und wurden entlang von Transekten aufgenommen, die das ganze Quadrat möglichst repräsentativ abdecken (Koordinationsstelle BDM 2008). Der Umfang belief sich auf 226 Stichproben bei den Gefässpflanzen, 224 bei den Tagfaltern und 237 bei den Brutvögeln.

Auf der Landschaftsebene beschränkten wir unsere Analyse auf die Vielfalt von Arten mit Waldbindung. Bei den Gefässpflanzen berücksichtigten wir nur diejenigen Arten als Waldarten, bei denen 75\% und mehr der Nachweise in der Datenbank des nationalen Artenzentrums aus dem Wald stammen. Bei den Tagfaltern stützten wir uns auf eine europaweite Einteilung der Nachweise nach Habitattyp (van Swaay et al 2006). Bei den Vögeln analysierten wir sämtliche Arten, bei welchen der Wald oder waldähnliche Habitate als Lebensraum im Artprofil der Vogelwarte Sempach aufgelistet sind.

\section{Umweltdaten auf Bestandesebene}

Um den Standort und seinen Einfluss auf die Artenvielfalt möglichst umfassend zu beschreiben, verwendeten wir einen zu den Strukturparametern möglichst komplementären Satz von abiotischen und biotischen Umweltvariablen. Wir testeten verschiedene klimatische (z.B. Temperatur, Niederschlag) und topografische Variablen (z.B. topografische Position, topografischer Wasserfluss, Exposition), den pH-Wert des Oberbodens sowie den aus Satellitenbildern abgeleiteten Produktivitätsindex «Normalized Difference Vegetation Index» (NDVI; Zimmermann \& Kienast 1999, Zimmermann \& Roberts 2001, Zellweger et al 2015).

LiDAR-Daten verwendeten wir, um die Bestandesstruktur flächendeckend abzubilden. Die LiDAR-Daten liegen als Punktwolken vor, wobei unterschieden wird, ob ein Punkt von der Vegetation oder dem Boden stammt (Abbildung 1). Als Indikator für die Lichtverhältnisse am Boden haben wir den Anteil von Vegetationspunkten, d.h. LiDARPunkten auf über einem Meter Höhe, an allen LiDAR-Punkten (inkl. jener, die den Boden erreichten) berechnet. Insofern gleicht dieser Indikator einem Vegetationsdeckungsgrad, der beschreibt, wie viel Licht auf den Waldboden gelangt (Müller \& Brandl 2009). Um die vertikale Heterogenität der Vegetation zu beurteilen, haben wir die Standardabweichung der Vegetationshöhen berechnet. Sämt-

\footnotetext{
1 Allgemeine Methoden: www.biodiversitymonitoring.ch/de/ downloads/methoden.html (14.3.2017)

2 www.infospecies.ch (13.3.2017)
} 
a)

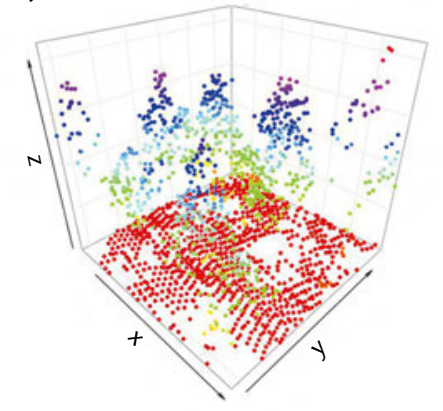

b)

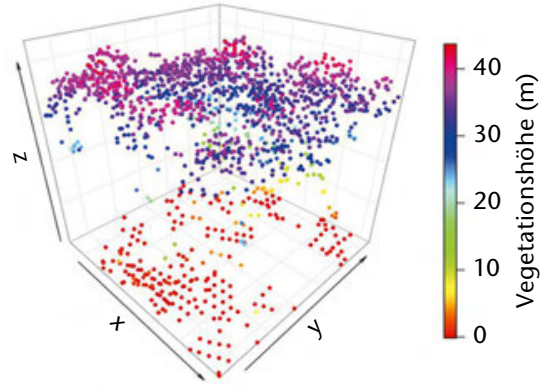

c)

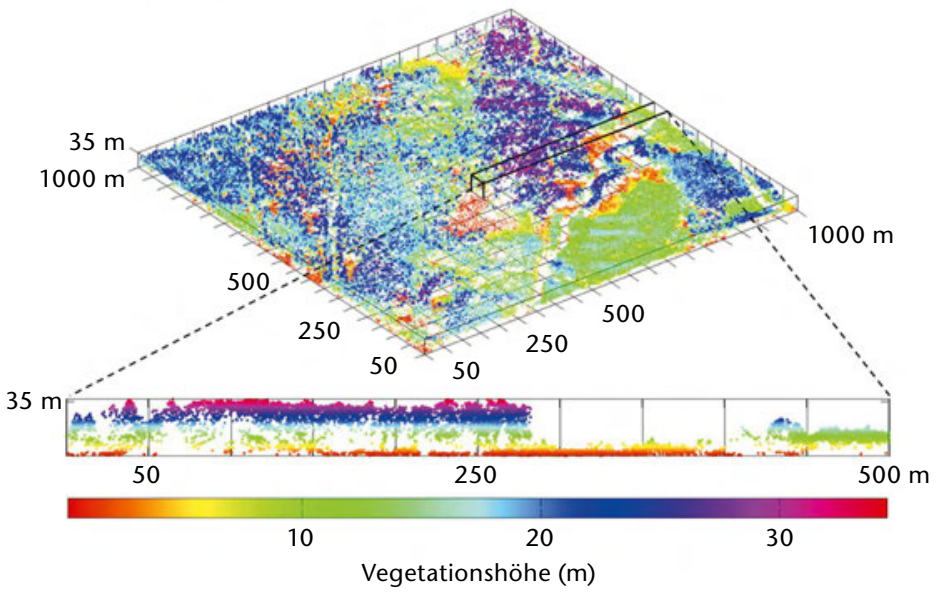

Abb 1 Geländekorrigierte, dreidimensionale Verteilung der LiDAR-Punkte im Wald. Die Abbildungen a) und b) zeigen die Struktur auf Bestandesebene $(30 \times 30 \mathrm{~m}), \mathrm{c})$ und d) diejenige auf Landschaftsebene $\left(1 \mathrm{~km}^{2}\right)$. a) Lockerer Fichtenbestand: Die Form einzelner Bäume ist deutlich sichtbar, und relativ viele Punkte erreichen die Erdoberfläche. b) Geschlossener Buchenhallenwald: Die Mehrheit aller Vegetationspunkte liegt im relativ dichten Kronendach. c) Bestandesmosaik auf einem bewaldeten Quadratkilometer. Die unterschiedlichen Vegetationshöhen treten deutlich hervor. d) Vertikale Verteilung der Vegetationspunkte entlang eines Transekts aus c).

liche Analysen auf Bestandesebene wurden mit einer räumlichen Auflösung (Rasterzellengrösse) von $30 \times 30 \mathrm{~m}$ durchgeführt.

Weiter berücksichtigten wir Bestandesdaten aus dem LFI, um Zielvariablen zu identifizieren, mit welchen die Habitatqualität im Bestand gefördert werden kann. Die ausgewählten Variablen beschreiben die Struktur, die Zusammensetzung und die Nutzung auf der Stichprobenfläche. Strukturelle Merkmale beinhalteten unter anderem die Grundfläche, den Deckungsgrad einzelner Schichten und der Bodenvegetation sowie das Volumen von stehendem und liegendem Totholz. Um die Zusammensetzung eines Bestandes zu beschreiben, analysierten wir den Mischungsgrad sowie den Grundflächenanteil von Baumarten der Gattungen Fraxinus, Tilia, Ulmus und Acer. Eine Eigenschaft dieser für Auenwälder typischen Baumarten besteht darin, dass ihre Blätter eine hohe Konzentration von löslichen Calciumsalzen aufweisen, welche von Landschnecken für den Aufbau des Gehäuses benötigt werden (Hotopp 2002). Informationen zur Waldnutzung beinhalteten unter anderem die Zeit seit dem letzten forstlichen Eingriff.

\section{Umweltdaten auf Landschaftsebene}

Ähnlich wie bei der Analyse auf Bestandesebene verwendeten wir auch für jene auf der Landschaftsebene eine breit abgestützte Liste von Umweltvariablen. Interpolierte klimatische Parameter lagen in einer Rasterzellengrösse von $100 \times 100$ m vor und beinhalteten unter anderem die Anzahl Gradtage (Wärmesumme; korreliert stark mit der Temperatur), den Niederschlag während der Vegetationszeit (April bis September), die jährliche Anzahl Frosttage und die potenzielle Sonneneinstrahlung. Topografische und edaphische Parameter $(25 \times 25$ m Rasterzellengrösse) umfassten die topografische Position, die Hangneigung und den Oberboden-pH. Die Waldstruktur wurde wiederum anhand von flächendeckend vorhandenen LiDAR-Daten hergeleitet und mit einer Rasterzellengrösse von $20 \times 20 \mathrm{~m}$ berechnet. Diese Parameter umfassten die Kronendachhöhe, die mittlere Vegetationshöhe, die Vegetationsdichte und ein Mass für die Heterogenität in der Unterschicht.

Um den Zusammenhang mit der Artenvielfalt auf den $1 \mathrm{~km}^{2}$ grossen Flächen zu untersuchen, wurden für alle Umweltdaten der Mittelwert und die Standardabweichung pro Quadratkilometer berechnet. Die Standardabweichung beschreibt somit die räumliche Heterogenität innerhalb eines Quadratkilometers.

\section{Datenanalyse und räumliche Vorhersage}

Das übergeordnete Ziel bestand darin, den Zusammenhang zwischen den Umweltvariablen und der Artenvielfalt pro Artengruppe statistisch zu untersuchen. $\mathrm{Zu}$ diesem Zweck wurden verschiedene Regressionstechniken verwendet. Auf der Bestandesebene wurden zwei Hauptanalysen durchgeführt. Einerseits analysierten wir den Einfluss der flächendeckend vorhanden Umweltdaten auf die Artenvielfalt an Gefässpflanzen, Moosen und Landschnecken mit dem Algorithmus RandomForest (Breiman 2001). Die daraus resultierenden Modelle wurden anhand des Prozentsatzes der erklärten Varianz und der relativen Wichtigkeit der einzelnen Variablen evaluiert. Anschliessend wurden die Modelle für die räumlich explizite Vorhersage der Artenvielfalt in einer Region in der Westschweiz verwendet. Andererseits untersuchten wir den Einfluss der LFI-Variablen, die nur auf den Stichprobenflächen vorlagen und somit nicht in die räumlich explizite Vorhersage integriert werden konnten, auf die Artenvielfalt anhand eines generalisierten linearen Modells (GLM), wobei vorgängig eine Variablenselektion mit dem LASSO-Algorithmus (Tibshirani 1996) durchgeführt wurde. Da wir standardisierte Variablenwerte verwendeten, können die Koeffizienten der kontinuierlichen Variablen als relative Wichtigkeit interpretiert werden. 


\begin{tabular}{|c|c|c|}
\hline \multirow[t]{2}{*}{ Artengruppe/Prädiktor } & \multicolumn{2}{|c|}{ Artenvielfalt } \\
\hline & Alle Arten & Waldarten \\
\hline \multicolumn{3}{|l|}{ Gefässpflanzen } \\
\hline $\mathrm{R}^{2}(\mathrm{SD})$ & $16.8(1.8)$ & \\
\hline Variable & Varlmp & \\
\hline Temperatur & $14.4(-)$ & \\
\hline Topografische Position & $6.2(-)$ & \\
\hline Östliche Exposition & $6.4(+)$ & \\
\hline $\mathrm{pH}$ & $8.1(+)$ & \\
\hline NDVI & $6.3(\cap)$ & \\
\hline Vegetationsdeckungsgrad & $7.7(-)$ & \\
\hline \multicolumn{3}{|l|}{ Moose } \\
\hline $\mathrm{R}^{2}(\mathrm{SD})$ & $24.0(3.0)$ & $20.3(2.3)$ \\
\hline Variable & Varlmp & Varlmp \\
\hline Temperatur & $16.3(-)$ & $10.2(-)$ \\
\hline Niederschlag & & $7.3(\cap)$ \\
\hline Standortwasserbilanz & $6.4(+)$ & $8.6(+)$ \\
\hline Sonneneinstrahlung & $10.0(-)$ & $8.4(-)$ \\
\hline Topografische Position & $7.3(-)$ & \\
\hline Topografischer Wasserfluss & $6.4(+)$ & \\
\hline $\mathrm{pH}$ & $6.1(b)$ & \\
\hline NDVI & & $8.3(+)$ \\
\hline Vegetationsdeckungsgrad & & $7.9(+)$ \\
\hline \multicolumn{3}{|l|}{ Landschnecken } \\
\hline $\mathrm{R}^{2}(\mathrm{SD})$ & $31.6(2.2)$ & $37.2(1.4)$ \\
\hline Variable & Varlmp & Varlmp \\
\hline Temperatur & $10.6(+)$ & $14.5(+)$ \\
\hline Niederschlag & $9.0(-)$ & $9.7(-)$ \\
\hline Standortwasserbilanz & $5.8(-)$ & $5.3(-)$ \\
\hline Topografischer Wasserfluss & $5.6(+)$ & \\
\hline $\mathrm{pH}$ & $18.0(+)$ & $16.1(+)$ \\
\hline NDVI & & $5.6(+)$ \\
\hline Standardabweichung Vegetationshöhe & $7.6(+)$ & $7.7(+)$ \\
\hline
\end{tabular}

Tab 1 Resultate der Modelle für Artenvielfalt (alle Arten, Waldarten) pro Artengruppe auf Bestandesebene, basierend auf den flächendeckend vorhandenen Umweltprädiktoren. $R^{2}$ : Anteil erklärter Varianz. Varlmp (\%): Relative Wichtigkeit der Variablen, ein Wert ist für die sechs jeweils wichtigsten Prädiktoren angegeben. Die Richtung des Zusammenhangs (+: positiv, -: negativ, $\cap$ : buckelförmig) zwischen der Artenvielfalt und einem Umweltprädiktor basiert auf einer visuellen Einschätzung der Reaktionskurve aus den Modellen. Aufgrund der niedrigen Modellgüte wurde bei den Gefässpflanzen kein Modell für die Vielfalt an Waldarten aufgelistet. Erläuterungen zu einzelnen Umweltvariablen: Die topografische Position beschreibt, ob der Standort eher auf einer Kuppe oder in einer Senke liegt. Der topografische Wasserfluss ist ein Mass für den seitlichen Wasserfluss, die Standortwasserbilanz ein Mass für Feuchtigkeit. Der Normalized Difference Vegetation Index (NDVI) ist ein Produktivitätsindex und die Standardabweichung von der Vegetationshöhe ein Mass für die vertikale Heterogenität.

Auf der Landschaftsebene führten wir eine aus mehreren Algorithmen bestehende Regressionsanalyse durch. Die Modelle wurden ebenfalls anhand des Prozentsatzes der erklärten Varianz sowie der relativen Wichtigkeit der Variablen evaluiert. Für jede Variable wurden die Art und die Richtung des Zusammenhangs visuell aus den Reaktionskurven abgeleitet.

\section{Ergebnisse}

\section{Artenvielfalt auf Bestandesebene}

Insgesamt untersuchten wir auf Bestandesebene 749 Gefässpflanzenarten, 336 Moosarten und 117 Landschneckenarten, wobei jeweils rund die Hälfte der Arten als Waldarten klassiert wurde (Zellweger et al 2015). Der Anteil an erklärter Varianz in den Modellen mit den flächendeckend vorhandenen Umweltdaten variierte zwischen $37.2 \%$ und $16.8 \%$, wobei für die Artenvielfalt von Waldgefässpflanzen kein Modell präsentiert werden kann, da hier nur ein schwacher statistischer Zusammenhang bestand (Tabelle 1). Die Artenvielfalt der Landschnecken konnte am besten vorhergesagt werden, gefolgt von derjenigen der Moose und der Gefässpflanzen. Die Vielfalt der letzten zwei Artengruppen nahm mit abnehmender Temperatur zu, während jene der Landschnecken mit zunehmender Temperatur anstieg. Die Artenvielfalt nahm bei den Gefässpflanzen und insbesondere bei den Landschnecken mit zunehmendem Oberboden-pH zu. Ein abnehmender Vegetationsdeckungsgrad hatte einen positiven Einfluss auf die Artenvielfalt bei den Gefässpflanzen und einen negativen bei den Waldmoosen. Mit zunehmender vertikaler Heterogenität des Waldes (Standardabweichung der Vegetationshöhe) nahm die Anzahl Landschneckenarten zu. Generell gleichen sich die für eine Artengruppe und die entsprechende Waldartengruppe gefundenen Beziehungen in Richtung und Stärke.

Die flächendeckenden räumlichen Vorhersagen für eine Beispielregion in der Westschweiz zeigen ein artengruppenspezifisches Muster (Abbildung 2). Während die vorhergesagte Verbreitung der Artenvielfalt für Gefässpflanzen und Moose ähnlich ist, ist jene der Landschnecken weitgehend komplementär zu den anderen beiden Artengruppen.

\section{Artenvielfalt in Abhängigkeit von LFI-Variablen auf Bestandesebene}

Vor allem die Artenvielfalt von (Wald-)Landschnecken lässt sich mit den berücksichtigten LFIVariablen erklären (Tabelle 2): Diese nahm mit zunehmender Grundfläche typischer Auenbaumarten (Fraxinus, Tilia, Ulmus und Acer spp.) sowie mit steigendem Deckungsgrad sowohl der Ober- als auch der Unterschicht zu. Bei den Waldlandschnecken, nicht aber bei allen Landschnecken, stieg die Artenvielfalt zudem mit zunehmendem Totholzvolumen. In reinen Nadelholzbeständen ist die Artenvielfalt von Landschnecken geringer. Während die Vielfalt an Gefässpflanzenarten mit abnehmendem Deckungsgrad der Oberschicht stark zunahm, war dieser Zusammenhang bei den Waldgefässpflanzen deutlich weniger ausgeprägt. Die Artenvielfalt bei den Moosen und den Waldmoosen konnte mit den hier getesteten LFI-Variablen nicht erklärt werden. 


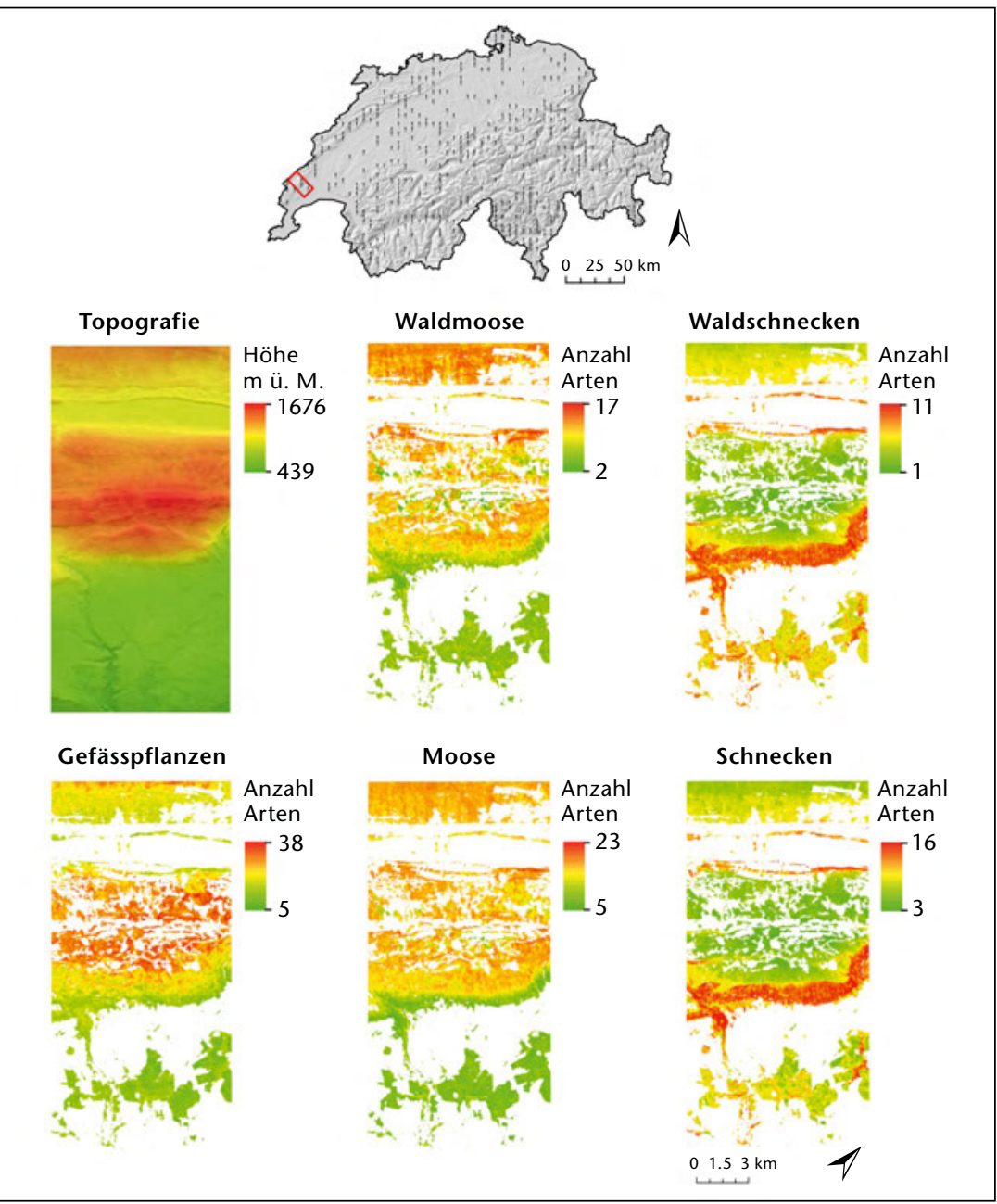

Abb 2 Räumliche Vorhersage der Artenvielfalt auf Bestandesebene pro Artengruppe, d.h. der Anzahl Arten pro Waldrasterzelle $(30 \times 30 \mathrm{~m})$ im Studiengebiet in der Romandie. Die Schweizer Karte zeigt die Stichprobenverteilung für die räumliche Vorhersage sowie die Lage des Studiengebiets.

\begin{tabular}{|c|c|c|}
\hline \multirow[t]{2}{*}{ Artengruppe/Prädiktor } & \multicolumn{2}{|c|}{ Artenvielfalt } \\
\hline & Alle Arten & Waldarten \\
\hline \multicolumn{3}{|l|}{ Gefässpflanzen } \\
\hline (Intercept) & $2.933^{* * *}$ & $2.599 * * *$ \\
\hline Deckungsgrad Oberschicht & $-0.205^{\star * *}$ & $-0.085^{\star *}$ \\
\hline Deckungsgrad Unterschicht & & $0.071^{* *}$ \\
\hline Grundfläche typischer Auenbaumarten & & $0.072 * *$ \\
\hline \multicolumn{3}{|l|}{ Landschnecken } \\
\hline (Intercept) & $2.054^{* * *}$ & $1.605^{* * *}$ \\
\hline Deckungsgrad Oberschicht & $0.144^{* *}$ & $0.188^{* * *}$ \\
\hline Deckungsgrad Unterschicht & $0.077^{*}$ & $0.097^{* *}$ \\
\hline $\begin{array}{l}\text { Deckungsgrad der Bodenvegetation } \\
76-100 \% \text { (kat.) }\end{array}$ & & $-0.324^{* *}$ \\
\hline Nadelholz rein $(91-100 \%$, kat.) & $-0.294^{* * *}$ & $-0.310^{* * *}$ \\
\hline Grundfläche & $-0.114^{* *}$ & $-0.138^{* *}$ \\
\hline Grundfläche typischer Auenbaumarten & $0.154^{* * *}$ & $0.171^{* * *}$ \\
\hline Totholzvolumen (stehend und liegend) & & $0.110 * \star$ \\
\hline Zeit seit letztem forstlichem Eingriff & $-0.144^{\star * *}$ & $-0.193^{* * *}$ \\
\hline
\end{tabular}

Tab 2 Koeffizienten und Signifikanzniveaus von generalisierten linearen Modellen, mit welchen die Artenvielfalt mithilfe von LFI-Variablen modelliert wurde. Da die Variablen standardisiert wurden, können die Koeffizienten als relative Wichtigkeit der Variablen interpretiert werden (ausser kategoriale Variablen [kat.]). Signifikanzniveaus: *** 0.001, ** 0.01, *0.05. Für Moose konnte wegen fehlenden Zusammenhangs mit den getesteten Variablen kein Modell gerechnet werden.

\section{Artenvielfalt auf Landschaftsebene}

Auf der Landschaftsebene haben wir 212 Waldgefässpflanzen sowie 157 Tagfalter- und 92 Vogelarten mit Waldbindung untersucht (Zellweger et al 2016). Der Anteil an erklärter Varianz war am höchsten bei den Modellen für Waldgefässpflanzen (67.2\%), gefolgt von denjenigen für Tagfalter (54.2\%) und Vögel (44.1\%; Tabelle 3). Die Anzahl Gradtage hatte bei den Waldgefässpflanzen mit Abstand den stärksten Einfluss auf die Artenvielfalt. Diese nahm mit zunehmender Anzahl Gradtage zu. Die mittlere Vegetationshöhe und der Oberboden-pH hatten ebenfalls einen positiven Effekt. Die Artenvielfalt bei den Tagfalterarten stieg mit zunehmender Heterogenität in der Unterschicht sowie mit zunehmender Hangneigung an. Bei den Vögeln ergaben sich weniger deutliche Zusammenhänge, wobei sich die Artenvielfalt mit zunehmendem Niederschlag und zunehmender räumlicher Variabilität der Anzahl Frosttage verkleinerte. Weiter bestand ein positiver Zusammenhang zwischen der Artenvielfalt der Vögel und flachem oder leicht kuppenförmigem Relief, zunehmender Sonneneinstrahlung sowie abnehmender Vegetationsdichte (Tabelle 3).

Die räumlichen Vorhersagen für alle Quadratkilometer der Schweiz, die zu mehr als 30\% bewaldet sind, zeigen ebenfalls artengruppenspezifische Muster (Abbildung 3): Bei den Waldgefässpflanzen wird eine hohe Artenvielfalt für die Föhntäler, die tieferen Lagen des Juras sowie für Teile des Mittellandes vorhergesagt. Hier ist es wichtig, zu berücksichtigen, dass es sich um Gefässpflanzen mit starker Bindung an den Wald handelt. Die höchste Artenvielfalt bei den Tagfaltern wird für die inneralpinen Täler und insbesondere für das Wallis vorhergesagt. Gemäss dem Modell für Vögel ist die Artenvielfalt im Mittelland sowie in tieferen und oft südexponierten Lagen der Bergregionen auf der Alpennordseite am höchsten.

\section{Diskussion}

Neben klimatischen und edaphischen Faktoren ist die Waldstruktur sowohl auf der Bestandesebene als auch auf der Landschaftsebene von Bedeutung für die Artenvielfalt. Sie beeinflusst die Habitat- und Standortqualität für Pflanzen und Moose sowie für die wenig mobilen Landschnecken, indem sie unter anderem die mikroklimatischen Verhältnisse am Waldboden mitprägt. Eine zunehmende Beschattung durch die Strauch- und Baumschicht beispielsweise wirkt sich positiv auf das Vorkommen vieler Landschneckenarten aus, weil dadurch das Austrocknen der Waldbodenoberfläche reduziert wird und starke mikroklimatische Schwankungen ausbleiben (Kappes 2013). Umgekehrt ist die Verbreitung vieler Pflanzenarten durch 


\section{Waldgefässpflanzen}

\begin{tabular}{|l|c|}
\hline $\mathrm{R}^{2}(\mathrm{SD})$ & $67.2(5.1)$ \\
\hline Variable & Varlmp \\
\hline Anzahl Gradtage (Wärmesumme) & $0.67(+)$ \\
\hline Mittlere Vegetationshöhe & $0.10(+)$ \\
\hline pH-Wert des Oberbodens & $0.07(+)$ \\
\hline Standardabweichung topografische Position & $0.02(+)$ \\
\hline Standardabweichung Anzahl Gradtage (Wärmesumme) & $0.02(\cap)$ \\
\hline Hangneigung & $0.01(+)$ \\
\hline Tagfalter & \\
\hline R2 (SD) & $54.2(6.9)$ \\
\hline Variable & Varlmp \\
\hline Heterogenität in der Unterschicht & $0.25(+)$ \\
\hline Hangneigung & $0.22(+)$ \\
\hline Anzahl Gradtage (Wärmesumme) & $0.03(-)$ \\
\hline Standardabweichung Anzahl Gradtage (Wärmesumme) & $0.02(\cap)$ \\
\hline pH-Wert des Oberbodens & $0.02(\cap)$ \\
\hline Sonneneinstrahlung & $0.01(+)$ \\
\hline Vögel & \\
\hline R2 (SD) & $0.14 .1(5.6)$ \\
\hline Variable & Varlmp \\
\hline Niederschlag & $0.26(-)$ \\
\hline Standardabweichung Anzahl Frosttage & $0.17(-)$ \\
\hline Topografische Position & $0.08(\cap)$ \\
\hline Sonneneinstrahlung & $0.07(+)$ \\
\hline Vegetationsdichte & $0.06(-)$ \\
\hline Heterogenität in der Unterschicht & $0.04(\cap)$ \\
\hline Tab Resulteter & \\
\hline
\end{tabular}

Tab 3 Resultate der Modelle für Artenvielfalt (Waldarten) pro Artengruppe auf der Landschaftsebene. R$^{2}$ : Anteil erklärter Varianz. Varlmp: Relative Wichtigkeit der Variablen, ein Wert ist für die sechs jeweils wichtigsten Prädiktoren angegeben. Die Richtung des Zusammenhangs (+: positiv, -: negativ, $\cap$ : buckelförmig) zwischen der Artenvielfalt und einem Umweltprädiktor basiert auf einer visuellen Einschätzung der Reaktionskurve aus den Modellen. Standardabweichungen sind ein Mass für die räumliche Heterogenität der jeweiligen Umweltvariable innerhalb eines Quadratkilometers. die Lichtverfügbarkeit im Bodenbereich beeinflusst, was dazu führt, dass die Standortqualität für viele lichtbedürftige Gefässpflanzen mit zunehmender Beschattung durch die Baumschicht abnimmt. Dies wird auch durch Studien bestätigt, die genutzte Buchenwälder mit Buchen-Naturwaldreservaten verglichen (Schmidt 2005). Gemäss Kriebitzsch et al (2013) benötigt die grösste Gruppe der gefährdeten Gefässpflanzen und bodenbewohnenden Moose des Waldes eine Kombination aus nährstoffarmen Böden und offenem Kronendach.

Auf der Landschaftsebene war der Einfluss der Waldstruktur auf die Artenvielfalt bei den Tagfaltern am deutlichsten erkennbar, wobei mit zunehmender Heterogenität in der Unterschicht eine steigende Anzahl Arten beobachtet wurde. Gerade in lichten Wäldern und entlang von reichhaltig strukturierten Waldrändern dürfte unser aus LiDAR abgeleitetes Mass für Heterogenität in der Unterschicht hoch sein. Eine hohe Heterogenität in der Unterschicht wirkt sich positiv auf das Vorkommen vieler Tagfalterarten aus, weil solche Habitate eine Vielzahl von verschiedenen Habitatstrukturen enthalten. Beispiele solcher Elemente sind das Angebot von Schutz und Deckung vor Feinden, vor schlechtem Wetter oder menschlichen Störungen (z.B. Mähen), aber auch von geeigneten Eiablageplätzen (Dover et al 1997, Bollmann et al 2009). Weiter zeichnen sich solche Habitate durch ein relativ gutes Angebot an Nahrungspflanzen in der Form von Blütenpflanzen und Weichhölzern aus. Unsere Resultate bestätigen somit die verbreitete Meinung, dass strukturreiche Wälder eine Vielzahl von Nischen und strukturellen Habitatelementen bieten, die für das Überleben von vielen Arten wichtig sind (Flückiger \& Duelli 1997, Wermelinger et al 2007).
Abb 3 Vorhergesagte Artenvielfalt von Waldarten pro Artengruppe auf Landschaftsebene. Das Höhenmodell zeigt auch die Verteilung der verwendeten BDM-Stichproben (rote Punkte; mind. 30\% Waldanteil auf den Stichprobenquadraten von $1 \mathrm{~km}^{2}$ Grösse).

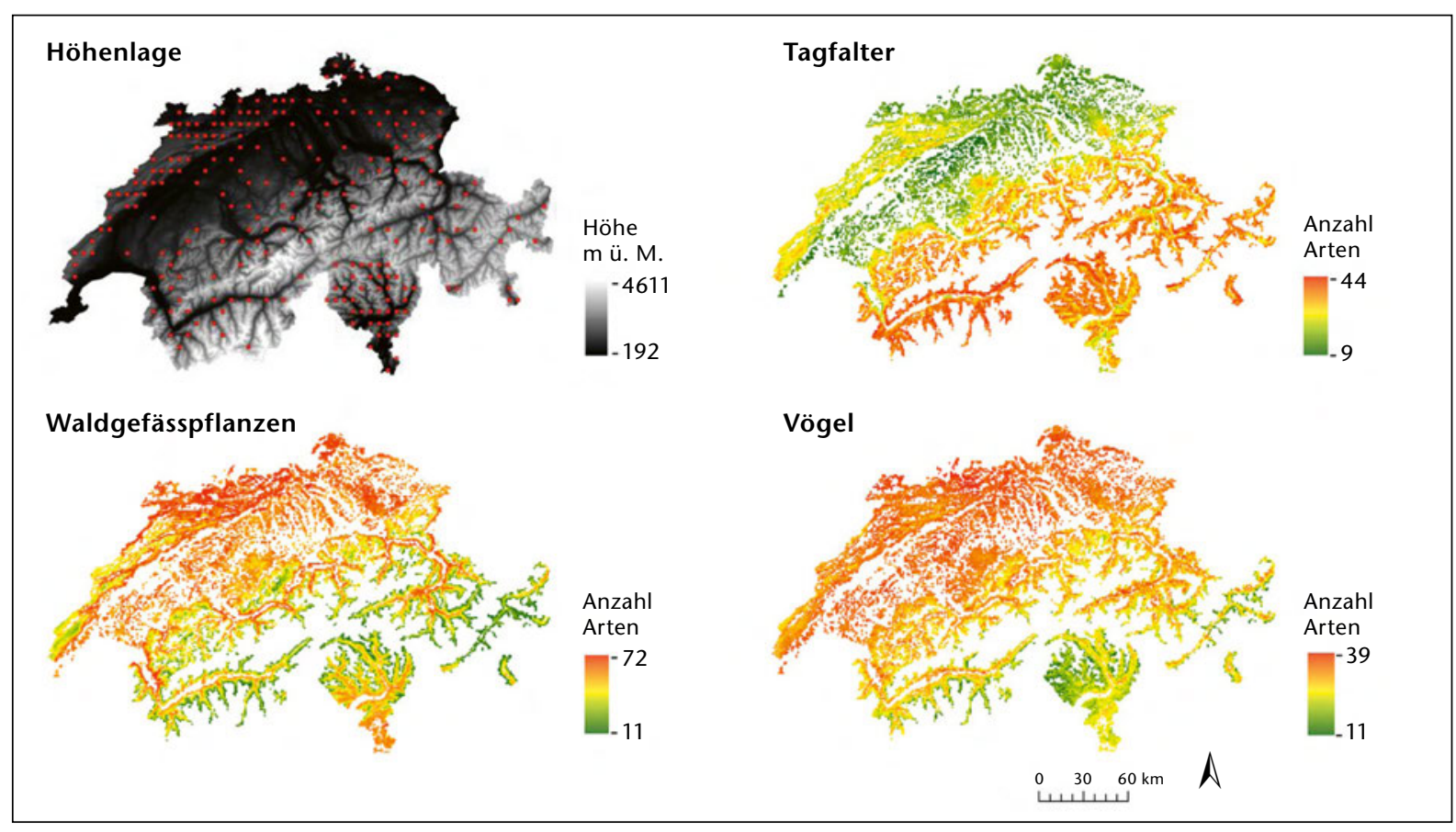


Die Güte der Modelle für die räumlichen Vorhersagen der Artenvielfalt war auf der Landschaftsebene höher als auf der Bestandesebene. Einer der Gründe für diesen Unterschied dürfte darin liegen, dass die für die Bestandesebene verwendeten Arterhebungen auf relativ kleinen Flächen $\left(10 \mathrm{~m}^{2}\right)$ durchgeführt werden. Für solche Untersuchungsflächen können die Standorteigenschaften mit den zur Verfügung stehenden Daten nicht genügend abgebildet werden, was das Erkennen von ökologischen Mustern erschwert. Nichtsdestotrotz war es möglich, auch auf Bestandesebene ökologisch sinnvolle Modelle zu berechnen. Insbesondere auf der Waldstruktur basierende räumliche Vorhersagen waren bis anhin nicht möglich. Wie von Davies \& Asner (2014) festgehalten, trägt die wachsende Verfügbarkeit von flächendeckenden LiDAR-Daten somit zur Förderung unseres Verständnisses von räumlichen Mustern der Artenvielfalt im Wald bei. LiDAR ist jedoch nicht die einzige Informationsquelle. Das nationale, aus Stereoluftbildern gewonnene Vegetationshöhenmodell liefert ebenfalls genaue Informationen zur Waldstruktur und hat den Vorteil, dass es in regelmässigen Zeitabständen erneuert wird (Ginzler \& Hobi 2016). Obwohl diese aus der Fernerkundung gewonnenen Waldstrukturdaten klaren Mehrwert bringen, sind sie nur begrenzt dazu geeignet, sämtliche für die Biodiversität wichtige Strukturparamter zu erfassen. Beispielsweise ist die Erkennung von Totholz sehr limitiert. Insofern wird es auch in Zukunft nötig sein, Fernerkundungsdaten mit im Feld gemessenen Strukturparametern, wie sie beispielsweise im LFI erhoben werden, zu kombinieren.

\section{Implikationen und Empfehlungen für die Waldbewirtschaftung}

Unsere Analysen haben ergeben, dass die Ansprüche an die Umweltbedingungen und insbesondere an die strukturellen Eigenschaften des Waldes artengruppenspezifisch sind. So unterscheiden sich etwa die Ansprüche von waldbewohnenden Landschnecken häufig von denen vieler waldassoziierter Insekten oder Gefässpflanzen, welche wärmere und trockenere Mikroklimabedingungen bevorzugen (Kappes 2013, diese Studie). Für eine umfassende und artengruppenübergreifende Förderung der Biodiversität im Wald gibt es daher kein einheitliches Rezept. Wichtig ist, den Bedürfnissen verschiedener Artengruppen Rechnung zu tragen und die Massnahmen räumlich zu priorisieren, um Zielkonflikte möglichst zu vermeiden (Bollmann 2011). Das übergeordnete Ziel sollte darin bestehen, ein räumlich und zeitlich vielfältiges Mosaik von Waldlebensräumen mit einer Vielfalt an strukturellen Eigenschaften zu fördern. Neben den Karten zur Artenvielfalt jeder Artengruppe, die wertvolle Informationen zur räumlichen
Evaluierung von Fördermassnahmen beisteuern, leiten wir aus unseren Resultaten die unten ausgeführten Empfehlungen für die Waldbewirtschaftung ab. Diese Aufstellung beschränkt sich auf die hier erarbeiteten Resultate und ist nicht als abschliessendes Dokument für eine integrative Förderung der Waldbiodiversität in der Forstpraxis zu verstehen (vgl. dazu Imesch et al 2015). Zudem muss berücksichtigt werden, dass in unserer Studie die generelle Artenvielfalt je Artengruppe (inklusive der Unterscheidung nach den Kategorien Waldarten - alle Arten) betrachtet und keine Differenzierung nach Zielarten des Naturschutzes vorgenommen wurde. Für Moose präsentieren wir zudem keine bestandesspezifischen Empfehlungen, weil sich solche aus den hier untersuchen LFI-Variablen nicht ableiten liessen. Die Resultate in Tabelle 1 weisen jedoch darauf hin, dass die Vielfalt an Moosen durch feuchte Waldstandorte mit geringer Sonneneinstrahlung gefördert wird.

- Gefässpflanzen: Ein lückiger Kronenschluss und die reichliche Verfügbarkeit von Licht am Waldboden wirken sich generell positiv auf die Artenvielfalt von Gefässpflanzen aus. Betrachtet man jedoch lediglich die Vielfalt an Waldarten, verringert sich die Stärke dieses Zusammenhangs, da viele dieser Arten relativ schattentolerant sind und eine kleinere Toleranz gegenüber Abweichungen vom Waldinnenklima zeigen (Kriebitzsch et al 2013, siehe auch Brändli et al 2007). Bestände mit einem relativ hohen Anteil an Baumarten der Gattungen Fraxinus, Tilia, Ulmus und Acer beherbergen tendenziell mehr Waldarten.

- Landschnecken: Waldlandschnecken reagieren sensibel auf die Qualität der Laubstreu und die mikroklimatischen Schwankungen im Oberboden (Martin \& Sommer 2004). Zudem zeigen unsere Resultate, dass sich reine Nadelholzbestände negativ auf die Vielfalt von Landschnecken auswirken. Generell gibt es mehr Arten in Beständen mit hohen Anteilen an Baumarten der Gattungen Fraxinus, Tilia, Ulmus und Acer, da deren Blätter eine hohe Konzentration von löslichen Calciumsalzen enthalten (Hotopp 2002). Eine gute Beschattung durch die Strauch- und Baumschicht wirkt sich positiv für viele Landschneckenarten aus, weil dadurch die Feuchtigkeitsverhältnisse am Waldboden verbessert werden (Kappes 2013). Weiter kann die Habitatqualität für Waldlandschnecken und andere wichtige Zersetzer (z.B. Landasseln, Tausendfüssler) durch mehr stehendes und liegendes Totholz gefördert werden (Kappes et al 2007). Neben der Erhaltung von Altholzbeständen sind aus den oben genannten Erkenntnissen abgeleitete Massnahmen an Standorten mit kalkreichen Böden und viel organischem Bodenmaterial sowie austretendem Grundwasser am vielversprechendsten (Martin \& Sommer 2004, Kappes et al 2012). 
- Tagfalter: Unsere Resultate auf der Landschaftsebene zeigen, dass viele Tagfalterarten von Waldbeständen profitieren, die durch eine reich strukturierte Unterschicht mit genügend Licht für Blütenpflanzen, Sträucher und Weichhölzer gekennzeichnet sind. Die Habitatqualität kann somit durch die Förderung dieser Elemente erhöht werden, zum Beispiel entlang von inneren und äusseren Waldrändern, um Waldriede und entlang von Waldbächen.

- Vögel: Obwohl die Resultate hier nicht gleich deutlich ausfielen wie bei den Tagfaltern, konnten wir zeigen, dass die Vielfalt an Vogelarten in Waldlandschaften mit lichten Beständen und mit mässig ausgeprägter Unterschicht tendenziell höher ist. Verstärkt wird dieser Effekt in Gebieten mit flachem oder leicht kuppenförmigem Relief und relativ hoher Sonneneinstrahlung. Bei der Interpretation dieser Resultate muss man berücksichtigen, dass die Vögel die mobilste Artengruppe der Studie darstellen und alle Arten berücksichtigt wurden, die mindestens teilweise auf den Wald als Lebensraum angewiesen sind.

\section{Schlussfolgerungen}

Aus LiDAR-Daten lassen sich flächendeckend Waldstrukturparameter ableiten. Damit verbessern sich unsere Möglichkeiten, Zusammenhänge zwischen Standorteigenschaften, Waldstruktur und Artenvielfalt zu erkennen und so die Effektivität von Massnahmen für den Erhalt und die Förderung der Biodiversität im Schweizer Wald zu verbessern. Die vorliegenden Resultate lassen den Schluss zu, dass die Ansprüche der untersuchten Artengruppen an die Umwelt und im Speziellen an die Waldstruktur artengruppenspezifisch sind. Dies spiegelt sich nicht zuletzt in der räumlichen Verteilung der Artenvielfalt im Schweizer Wald: Nur in wenigen Gebieten ist die Artenvielfalt für mehrere Artengruppen gleichzeitig hoch. Deshalb ist die Erhaltung von Gradienten für Bodenfeuchtigkeit, Licht, pH-Wert und Nährstoffe, wie sie unter natürlichen Bedingungen vorkommen, in Kombination mit der Förderung von Strukturvielfalt ein wichtiger Grundsatz bei der generellen Förderung von Waldarten. In Anbetracht der intensiven Bewirtschaftung des Kulturlands und der Nährstoffeinträge kommt dem Wald zusätzlich die Bedeutung als Rückzugsgebiet für gefährdete Arten mit einer breiten ökologische Nische zu (vgl. Bollmann \& Braunisch 2013).

Eingereicht: 2. August 2016, akzeptiert (mit Review): 27. März 2017

\section{Dank}

Wir bedanken uns beim Bundesamt für Umwelt für die Artdaten aus dem Biodiversitätsmonito- ring, welche uns von Hintermann \& Weber AG zur Verfügung gestellt wurden. F. Zellweger wurde vom Schweizerischen Nationalfonds zur Förderung der wissenschaftlichen Forschung finanziert (Projektnummer 146786).

\section{Literatur}

BOLLMANN K, BERGAMINI A, SENN-IRLET B, NOBIS M, DUELLI P ET AL (2009) Konzepte, Instrumente und Herausforderungen bei der Förderung der Biodiversität im Wald. Schweiz Z Forstwes 160: 53-67. doi: 10.3188/szf.2009.0053

BOLLMANN K (2011) Naturnaher Waldbau und Förderung der biologischen Vielfalt im Wald. In: WSL, Editor. Der multifunktionale Wald - Konflikte und Lösungen. Birmensdorf: Eidgenöss Forsch.anstalt WSL, Forum für Wissen 2011. pp. 27-36.

BOLLMANN K, BRAUNISCH V (2013) Integration oder Segregation: der Spagat zwischen der Produktion von Rohstoffen und dem Schutz der Biodiversität in europäischen Wäldern. In: Kraus D, Krumm F, editors. Integrative Ansätze als Chance für die Erhaltung der Artenvielfalt in Wäldern. Freiburg i. Br.: European Forest Institute. pp. 18-32.

BRÄNDLI UB, BÜHLER C, ZANGGER A (2007) Waldindikatoren zur Artenvielfalt: Erkenntnisse aus LFI und BDM. Schweiz Z Forstwes 158: 243-254. doi: 10.3188/szf.2007.0243

BREIMAN L (2001) Random Forests. Machine Learning 45: 5-32.

DAVIES AB, ASNER GP (2014) Advances in animal ecology from 3D-LiDAR ecosystem mapping. Trends Ecol Evol 29: 681-691.

DOVER JW, SPARKS TH, GREATOREX-DAVIES JN (1997) The importance of shelter for butterflies in open landscapes. J Insect Conserv 1: 89-97.

FLÜCKIGER PF, DUELLI P (1997) Forest edges - centres of biodiversity. Mitt Dtsch Ges allg angew Entomol 11: 119-123.

GACHET G, JUNOD P (2008) Apports et perspectives de la technologie LiDAR aéroporté pour la pratique forestière. J For Sui 159: 19-30. doi: 10.3188/szf.2008.0019

GINZLER C, HOBI ML (2016) Das aktuelle Vegetationshöhenmodell der Schweiz: spezifische Anwendungen im Waldbereich. Schweiz Z Forstwes 167: 128-135. doi: 10.3188/szf.2016.0128

GUÉNETTE JS, VILLARD MA (2005) Thresholds in forest bird response to habitat alteration as quantitative targets for conservation. Conserv Biol 19: 1168-1180.

HOTOPP KP (2002) Land snails and soil calcium in central Appalachian Mountain Forest. Southeastern Naturalist 1: 27-44.

HUNTER ML (1999) Maintaining biodiversity in forest ecosystems. Cambridge: Cambridge Univ Press. 698 p.

IMESCH N, STADLER B, BOLLIGER M, SCHNEIDER O (2015) Biodiversität im Wald: Ziele und Massnahmen. Vollzugshilfe zur Erhaltung und Förderung der biologischen Vielfalt im Schweizer Wald. Bern: Bundesamt Umwelt, Umwelt-Vollzug 1503. $186 \mathrm{p}$.

KAPPES H, CATALANO C, TOPP W (2007) Coarse woody debris ameliorates chemical and biotic soil parameters of acidified broad-leaved forests. Appl Soil Ecol 36: 190-198.

KAPPES H, CLAUSIUS A, TOPP W (2012) Historical small-scale surface structures as a model for post-mining land reclamation. Restor Ecol 20: 322-330.

KAPPES H (2013) Gehäuse- und Nacktschnecken als Indikatoren für nachhaltige Waldbewirtschaftung. In: Kraus D, Krumm F, editors. Integrative Ansätze als Chance für die Erhaltung der Artenvielfalt in Wäldern. Freiburg i. Br.: European Forest Institute. pp. 206-214.

KRIEBITZSCH WU, BÜLTMANN H, VON OHEIMB G, SCHMIDT M, THIEL H ET AL (2013) Waldspezifische Vielfalt der Gefässpflanzen, Moose und Flechten. In: Kraus D, Krumm F, editors. Integrative Ansätze als Chance für die Erhaltung der Artenvielfalt in Wäldern. Freiburg i. Br.: European Forest Institute. pp. 164-175. 
LINDENMAYER DB, FRANKLIN JF, FISCHER J (2006) General management principles and a checklist of strategies to guide forest biodiversity conservation. Biol Conserv 131: 433-445.

MARTIN K, SOMMER M (2004) Relationships between land snail assemblage patterns and soil properties in temperate-humid forest ecosystems. J Biogeogr 31: 531-545.

MACARTHUR R, MACARTHUR JW (1961) On bird species-diversity. Ecology 42: 594-598.

MORSDORF F (2011) Erfassung struktureller Waldparameter mithilfe von flugzeuggetragenem Laserscanning. Schweiz Z Forstwes 162: 164-170. doi: 10.3188/szf.2011.0164

MÜLLER J, BRANDL R (2009) Assessing biodiversity by remote sensing in mountainous terrain: the potential of LiDAR to predict forest beetle assemblages. J Appl Ecol 46: 897-905.

NASSET E (2002) Predicting forest stand characteristics with airborne scanning laser using a practical two-stage procedure and field data. Remote Sens Environ 80: 88-99.

RIGLING A, SCHAFFER HP, EDITORS (2015) Waldbericht 2015. Zustand und Nutzung des Schweizer Waldes. Bern: Bundesamt Umwelt. $143 \mathrm{p}$.

SCHMIDT W (2005) Herb layer species as indicator of biodiversity of managed and unmanaged beech forests. For Snow Landsc Res 79: 111-125.

SEIBOLD S, BRANDL R, BUSE J, HOTHORN T, SCHMIDL J ET AL (2015) Association of extinction risk of saproxylic beetles with ecological degradation of forests in Europe. Conserv Biol 29: 382-390.

TIBSHIRANI R (1996) Regression shrinkage and selection via the Lasso. J Royal Stat Soc, Series B (Methodological) 58: 267-288.

\section{La forêt suisse et sa biodiversité: LiDAR permet de nouvelles analyses de la structure forestière}

Des cartes de biodiversité et des recommandations spécifiques pour améliorer la qualité des habitats ne sont souvent pas disponibles pour le service forestier, bien que ces informations soient très utiles dans le cadre d'une gestion forestière multifonctionnelle. Dans cet article, nous combinons des paramètres de structure forestière dérivés des données nationales LiDAR avec des données du climat, de la topographie et du $\mathrm{pH}$ de sol, pour analyser et prédire spatialement la diversité des espèces en forêt selon deux échelles différentes. A échelle du peuplement $\left(900 \mathrm{~m}^{2}\right)$, nous avons analysé la diversité des plantes vasculaires, des bryophytes et des escargots terrestres et avons utilisé en même temps les données de I'Inventaire forestier national pour identifier des variables-clés pour améliorer I'habitat. A l'échelle du paysage $\left(1 \mathrm{~km}^{2}\right)$, nous avons analysé la diversité des plantes vasculaires, des papillons diurnes et des oiseaux nicheurs qui ont un fort lien avec la forêt. Les paramètres de la structure de la forêt selon LiDAR étaient d'importants prédicteurs de la diversité pour tous les genres. Les différents genres avaient des distributions spatiales différentes et leurs aires de répartition ne se recoupaient que partiellement. La diversité des escargots terrestres liés à la forêt augmente, par exemple, avec une plus forte proportion d'arbres du genre Fraxinus, Tilia, Ulmus et Acer, ou avec un degré de couverture et un volume de bois mort plus importants. La diversité des papillons diurnes, par contre, augmente avec I'hétérogèneité du sous-bois. Cette étude démontre que des caractéristiques structurelles pour la biodiversité forestière peuvent être relevées par LiDAR sur de grandes surfaces. La disponibilité croissante des données LiDAR fournit des informations utiles pour le maintien et la promotion de la biodiversité dans la sylviculture multifonctionnelle.
VAN SWAAY C, WARREN M, LOÏS G (2006) Biotope use and trends of European butterflies. J Ins Conserv 10: 189-209.

WEBER D, HINTERMANN U, ZANGGER A (2004) Scale and trends in species richness: considerations for monitoring biological diversity for political purposes. Glob Ecol Biogeogr 13: 97-104.

WERMELINGER B, FLÜCKIGER PF, OBRIST MK, DUELLI P (2007) Horizontal and vertical distribution of saproxylic beetles (Col., Buprestidae, Cerambycidae, Scolytinae) across sections of forest edges. J Appl Entomol 131: 104-114.

ZELLWEGER F, BRAUNISCH V, BALTENSWEILER A, BOLLMANN K (2013) Remotely sensed forest structural complexity predicts multi species occurrence at the landscape scale. For Ecol Manage 307: 303-312.

ZELLWEGER F, BRAUNISCH V, MORSDORF F, BALTENSWEILER A ABEGG M ET AL (2015) Disentangling the effects of climate, topography, soil and vegetation on stand-scale species richness in temperate forests. For Ecol Manage 349: 36-44.

ZELLWEGER F, BALTENSWEILER A, GINZLER C, ROTH T, BRAUNISCH V, BUGMANN H, BOLLMANN K (2016) Environmenta predictors of species richness in forest landscapes: abiotic factors versus vegetation structure. J Biogeogr 43: 1080-1090.

ZIMMERMANN NE, KIENAST F (1999) Predictive mapping of alpine grasslands in Switzerland: Species versus community approach. J Veg Sci 10: 469-482.

ZIMMERMANN NE, ROBERTS DW (2001) Final report of the MLP climate and biophysical mapping project. Birmensdorf: Swiss Federal Research Inst WSL. 9 p.

\section{The Swiss forest and its biodiversity: LiDAR allows for novel forest structure assessments}

Availability of comprehensive biodiversity maps and standlevel habitat management recommendations to forest services is often limited, despite the need for such information for multifunctional forestry. Here, we combined forest structural parameters derived from nation-wide available Light Detection and Ranging (LiDAR) data with parameters describing climate, topography and soil $\mathrm{pH}$, to analyse and spatially predict the species richness of different taxa on two different scales. Vascular plants, bryophytes and land snails were analysed on the stand scale $\left(900 \mathrm{~m}^{2}\right)$, where we also identified target variables for habitat management by using data from the National Forest Inventory. On the landscape scale $\left(1 \mathrm{~km}^{2}\right)$, we analysed vascular plants, butterflies and breeding birds with a tight association to forests. LiDAR-derived forest structure parameters were consistently important predictors of species richness across taxa. Species richness patterns tended to be taxon-specific with low spatial congruence across taxa. The habitat quality for many forest land snail species, for example, increased with increasing proportions of trees from the genera Fraxinus, Tilia, Ulmus and Acer, or with increasing overstory cover and deadwood volume. Many butterfly species, on the other hand, responded positively to a heterogeneous understory. This study shows that important structural parameters for forest biodiversity can be derived area-wide and across large regions by using LiDAR. The growing availability of LiDAR data thus provides very useful information for conserving and promoting biodiversity in multifunctional forestry. 